\title{
Electroweak boson production in the forward region with $\mathrm{LHCb}$
}

\author{
Tara SHEARS, on behalf of the LHCb Collaboration* \\ University of Liverpool \\ E-mail: tara.shearsecern.ch
}

\begin{abstract}
We have measured the $\mathrm{W}$ and $\mathrm{Z}$ boson production cross-sections in proton-proton collisions at a centre-of-mass energy of $7 \mathrm{TeV}$, using datasets of up to $240 \pm 8 \mathrm{pb}^{-1}$ collected by the $\mathrm{LHCb}$ experiment. All measurements are based on boson decays with at least one muon in the final state, which has transverse momentum $p_{T \mu}$ exceeding $20 \mathrm{GeV}$ and pseudorapidity $\eta_{\mu}$ within $2.0-4.5$. $\mathrm{Z}$ bosons are reconstructed in decays to muon or tau lepton pairs (the latter reconstructed in final states involving two muons, and one muon and one electron, within the same fiducial aceptance), where the reconstructed invariant mass $m_{Z}$ lies in the range $60 \leq m_{Z} \leq 120 \mathrm{GeV}$. In all cases results are consistent with next-to-next-to-leading order theoretical predictions.
\end{abstract}

XXIst International Europhysics Conference on High Energy Physics

21-27 July 2011

Grenoble, Rhône Alpes France

\footnotetext{
* Speaker.
} 


\section{Introduction}

Measurements of the $W$ and $Z^{1}$ cross-sections constitute an important test of the Standard Model in proton-proton collisions with a centre-of-mass energy of $7 \mathrm{TeV}$. Theoretical predictions have uncertainties of between $3 \%$ and $10 \%$, depending on rapidity, where the dominant uncertainty is due to knowledge of the parton distribution functions (PDFs) [1]. The LHCb experiment [2] is instrumented in the forward region with full tracking, calorimetry and particle identification capability between pseudorapidities of 1.9 to 4.9. Consequently, measurements made by LHCb can test the Standard Model and provide input to constrain the PDFs, both in the unique forward region $(\eta>2.5)$, and in a rapidity region which is in common to ATLAS and CMS $(2 \leq \eta \leq 2.5)$.

We report differential cross-section measurements and ratios of $W$ and $Z$ boson production. We present results for the total $W$ and $Z$ cross-sections inside our kinematic acceptance, where the final state muons have transverse momentum $p_{T \mu}$ exceeding $20 \mathrm{GeV}^{2}$ and lie within pseudorapidities of $2.0 \leq \eta_{\mu} \leq 4.5$, and, in the case of $Z$ production, an invariant mass $m_{Z}$ between $60 \leq m_{Z} \leq 120$ $\mathrm{GeV}$. These results are based on $37.1 \pm 1.3 \mathrm{pb}^{-1}$ of data. We also present results for the crosssection ratios $\sigma_{W} / \sigma_{Z}$ and $\sigma_{W+} / \sigma_{W-}$, and the $W$ charge asymmetry, $A_{W}$. In addition, we present measurements of $Z$ boson production in decays to pairs of tau leptons within the same kinematic acceptance, in final states containing two muons $(\mu \mu)$ and one muon and one electron $(\mu e)$, based on a dataset of $240 \pm 8 \mathrm{pb}$. Further information may be found in [3] and [4].

\section{Event selection}

All candidates are triggered by requiring one identified muon within the LHCb acceptance, with $p_{T}>20 \mathrm{GeV}$.

\section{$2.1 Z \rightarrow \mu \mu$}

Candidate $\mathrm{Z}$ events possess two well reconstructed muons which lie within the pseudorapidity range $2 \leq \eta \leq 4.5$, with $p_{T \mu}$ above $20 \mathrm{GeV}$. The invariant mass of the dimuon candidates, $M_{\mu \mu}$ must lie within $60 \leq M_{\mu \mu} \leq 120 \mathrm{GeV}$. 1966 candidates are selected.

The following background sources are considered:

(i) $Z \rightarrow \tau \tau$ where both taus decay to muons ( $0.61 \pm 0.04$ events, estimated from simulation);

(ii) $b \bar{b}$ and $c \bar{c}$ production ("heavy flavour"), with two semileptonic decays ( $4.3 \pm 1.7$ events, estimated using data events with high muon impact significance);

(iii) generic QCD events where pions or kaons either decay in flight or punch through the detector to be falsely identified as muons, either in pairs or in conjunction with a heavy flavour semileptonic decay ( $0 \pm 1$ events, determined from same sign dimuon combinations in data).

The total background estimate is taken as $4.9 \pm 2.0$ events.

\section{$2.2 W \rightarrow \mu v_{\mu}$}

Candidate $\mathrm{W}$ events possess one well reconstructed isolated muon candidate with a $p_{T \mu}$ greater than $20 \mathrm{GeV}$ within $2 \leq \eta \leq 4.5$. As backgrounds to the $W$ analysis are larger than for the $Z$ analysis

\footnotetext{
${ }^{1}$ In this paper, $Z$ refers to both $Z$ and $\gamma^{*}$ production around the $Z$ pole.

${ }^{2}$ Note that units are quoted throughout this note with $\mathrm{c}=1$.
} 
we impose additional criteria on muon identification and isolation, and ask that the muon should be consistent with the primary vertex.

Backgrounds from punch-through pions are suppressed by requiring that the sum of hadronic and electromagnetic calorimeter energies associated with the muon candidate, divided by the track momentum, is less than 0.04 . Isolation is imposed by requiring that the summed $p_{T}$ of all tracks and identified photons inside a cone of radius $r=\sqrt{\Delta \eta^{2}+\Delta \phi^{2}}=0.5$ surrounding the muon, $r_{\text {cone }}$, should be less than $2 \mathrm{GeV}$. The unbiased impact parameter, $I P$, of the muon must be less than $40 \mu \mathrm{m}$ to reduce backgrounds from heavy flavour production. Contributions from $\gamma^{*} / Z$ decay are suppressed by vetoing any other muon in the event with a $p_{T}$ above $5 \mathrm{GeV}$. After these requirements are made 15608 (12301) $W^{+}\left(W^{-}\right)$candidates are observed. The efficiency of the selection is estimated using $Z$ candidates in data, where one muon is removed to simulate a $W$ boson, and varies between $45 \%$ and $80 \%$ depending on lepton pseudorapidity.

The following background sources have been considered:

(i) $Z \rightarrow \mu \mu$ where one of the muons goes outside of the LHCb acceptance;

(ii) $W \rightarrow \tau \nu_{\tau}$ where the tau decays leptonically to a muon and neutrinos;

(iii) $Z \rightarrow \tau \tau$ where the tau decays leptonically to a muon and neutrinos;

(iv) Lower mass Drell-Yan production of dimuon final states, where one muon is outside the LHCb acceptance;

(v) b and c events containing semileptonic decays with a muon in the final state;

(vi) generic QCD events where pions or kaons decay in flight;

(vii) generic QCD events where pions or kaons punch through to give muons.

The signal yield is estimated by fitting the muon $p_{T}$ spectrum to the shapes expected for signal (obtained from next-to-leading order simulation, using POWHEG [5] and the CTEQ6m [6] parton density set) and each background class. In the fit, only the overall normalisations of the signal, and background (vi), are allowed to vary. The fit is performed for both charges and over all pseudorapidity bins simultaneously. The shapes for backgrounds (i) to (iv) are determined using simulation (POWHEG for (i), Pythia [7] otherwise). The normalisation of (i) is constrained to the observed rate of $Z$ production, and (ii) to the observed rate of $W$ production, taking branching ratios of $\tau$ to muons into account. The contributions of (iii) and (iv) are estimated from simulation. The shapes for backgrounds (v), (vi) and (vii) are found directly from data by anti-cutting on selection variables to enrich these sources. The normalisations are fixed to the rates observed in data, with the exception of decay in flight which is allowed to vary in the fit. The purity of the $W^{+}\left(W^{-}\right)$ sample is $80 \%(78 \%)$.

\section{$2.3 Z \rightarrow \tau \tau$}

Candidate events possess one well reconstructed isolated muon candidate with a $p_{T \mu}$ greater than $20 \mathrm{GeV}$ within $2 \leq \eta \leq 4.5$. The sum of hadronic and electromagnetic calorimeter energies associated with the muon candidate, divided by the track momentum, must be less than 0.2.

In the muon-electron final state and additional electron candidate with $p_{T}>5 \mathrm{GeV}$ is required. Electrons are identified within the same pseudorapidity region as muons, and must have energy deposits in the electromagnetic and hadronic calorimeters, and preshower detector consistent with electron deposition. The leptons must be isolated, and back-to-back in phi. 81 candidates pass the selection. In the dimuon final state the additional muon must have $p_{T}>5 \mathrm{GeV}$, and both 
leptons must be isolated and back-to-back. To suppress the higher Drell-Yan background in this channel the $I P$ significance must exceed 4 , and the $P_{T}$ asymmetry between both muons must exceed 0.2. 33 candidates pass the selection. The selection efficiencies for both selections are determined from simulation, and an uncertainty estimated from comparing agreement between distributions in simulation and data.

The following backgrounds have been considered:

(i) QCD, estimated from same-sign and non-isolated data $(9.5 \pm 3(1.6 \pm 1.3)$ for $\mu e(\mu \mu))$;

(ii) Electroweak; diboson and top backgrounds are estimated from simulation. Drell-Yan backgrounds in the dimuon channel are estimated from data, by fitting $M_{\mu \mu}$ in data above $80 \mathrm{GeV}$ to the expected shape from this contribution $(3 \pm 1.2(5.5 \pm 1.8)$ events in $\mu e(\mu \mu)$ channels.

\section{Cross-section measurement}

The cross-section, $\sigma_{Z}$, for $Z$ boson production in a rapidity region, $\Delta y$ can be written

$$
\sigma_{Z \rightarrow \mu \mu}(\Delta y)=\frac{N_{t o t}^{Z}-N_{b k g}^{Z}}{\varepsilon_{Z} L},
$$

where $N_{\text {tot }}^{Z}$ is the number of selected events, $N_{b k g}^{Z}$ is the estimated number of background events, $L$ is the integrated luminosity corresponding to the dataset used for the analysis, and $\varepsilon_{Z}$ is the overall efficiency for selecting signal events. This efficiency can be expressed as a product of: an acceptance factor, $A^{Z}$, which is the proportion of events that should be reconstructed inside the given kinematic region; a trigger efficiency, $\varepsilon_{\text {trig }}^{Z}$, which is the probability of triggering on such an offline selected event; a tracking efficiency, $\varepsilon_{\text {track }}^{Z}$, which is the probability of reconstructing both $Z$ decay products as tracks; a lepton reconstruction efficiency, $\varepsilon_{\text {muon }}^{Z}$, which is the probability of identifying both tracks as leptons; and a selection efficiency, $\varepsilon_{\text {sel }}^{Z}$, which is the probability of these leptons passing additional selection cuts.

Similarly, we express the cross-section for $W$ production, $\sigma_{W}$, in a muon pseudorapidity interval $\Delta \eta$ as

$$
\sigma_{W \rightarrow \mu v}(\Delta \eta)=\frac{N_{t o t}^{W}-N_{b k g}^{W}}{\varepsilon_{W} L},
$$

where now $\varepsilon_{W}=A^{W} \varepsilon_{\text {trig }}^{W} \varepsilon_{\text {track }}^{W} \varepsilon_{\text {muon }}^{W} \varepsilon_{\text {sel }}^{W}$, using the same notation as above, but with a $W$ superscript or subscript in place of a $Z$ and where the efficiencies correspond to one, and not two muons.

It should be noted that all cross-sections are quoted within the fiducial region we measure, and are not corrected to the full $4 \pi$ coverage. Cross-sections are corrected for final state radiation (FSR) to allow comparison with theory. Corrections are calculated using the HORACE [8] generator.

\subsection{Efficiencies}

Muon trigger, muon identification and muon tracking efficiencies are determined directly from data. A tag and probe technique is adopted in $Z \rightarrow \mu \mu$ events, where one muon (the tag) is well identified and fires the single muon trigger, and the other muon or track candidate (the probe) is tested to see how often it also fires the muon trigger, is identified as a muon, or has a track associated to it, depending on the efficiency under test. Efficiencies are determined as a function of lepton pseudorapidity. No dependance on lepton charge is observed. 
Electron tracking efficiencies are estimated from simulation, and scaled by the difference in tracking efficiency observed between simulation and data for muons. The electron identification efficiency is determined from data using a tag and probe method in $Z \rightarrow e e$ events.

\subsection{Systematic errors}

Systematic errors arise from: backgrounds; efficiencies; the FSR correction applied to the cross-section; the luminosity estimate. The uncertainties on backgrounds arise from Monte Carlo statistics and/or available statistics in data. The uncertainty in efficiency is taken as the statistical precision of the efficiency determination in data, and encompasses differences between data and simulation where calculated on Monte Carlo data. The FSR correction uncertainty is given by the statistical uncertainty on the HORACE estimate. The luminosity is determined by two methods, a Van Der Meer scan, and a beam gas method [9]. Both methods give similar results and are estimated to have a precision of order $3.5 \%$. This is the leading cause of systematic uncertainty for $W$ and $Z$ production in the muon channels. For $Z$ production of tau leptons, the efficiency uncertainty dominates. All the systematic error sources are combined in quadrature. They are summarised (as percentages), for the total measured $W$ and $Z$ cross-sections in table 1 .

\begin{tabular}{|c|c|c|c|c|c|}
\hline Source & $\Delta \sigma\left(\mathrm{W}^{+}\right)(\%)$ & $\Delta \sigma\left(\mathrm{W}^{-}\right)(\%)$ & $\Delta \sigma(\mathrm{Z})(\%)$ & $\Delta \sigma(e \mu)(\%)$ & $\Delta \sigma(\mu \mu)(\%)$ \\
\hline Backgrounds & \pm 1.6 & \pm 1.6 & \pm 0.4 & \pm 5 & \pm 7 \\
Template shape (fit) & \pm 1.9 & \pm 1.7 & $\mathrm{n} / \mathrm{a}$ & $\mathrm{n} / \mathrm{a}$ & $\mathrm{n} / \mathrm{a}$ \\
Efficiency & \pm 2.5 & \pm 2.3 & \pm 5.1 & \pm 9 & \pm 9 \\
FSR correction & \pm 0.2 & \pm 0.2 & \pm 0.3 & \pm 0.3 & \pm 0.3 \\
\hline Total systematic error & \pm 3.5 & \pm 3.2 & \pm 5.1 & \pm 10 & \pm 11 \\
\hline Luminosity & \pm 3.5 & \pm 3.5 & \pm 3.5 & \pm 3.5 & \pm 3.5 \\
\hline
\end{tabular}

Table 1: Contributions to the systematic error for the total $W$ and $Z$ cross-sections. Note that $e \mu$ and $\mu \mu$ refer to the final states for $Z \rightarrow \tau \tau$ production. All values are quoted as percentages. The luminosity uncertainty is quoted separately.

\subsection{Results}

The cross-sections of $W$ and $Z$ production within the $\mathrm{LHCb}$ fiducial are shown in figure 1 (left), together with the measured $\mathrm{W}$ charge asymmetry (right). The measurements are shown as a coloured band on the summary and are compared to various predictions based on next-to-leading order and next-to-next-to-leading order calculations (NNLO) and a variety of PDF sets. The W charge asymmetry is shown as data points, compared to NNLO prediction. It can be seen that all results are consistent with theoretical prediction.

\section{Conclusions}

The cross-sections and ratios of $W$ and $Z$ bosons have been measured using $37.1 \mathrm{pb}^{-1}(240 \pm 8$ $\mathrm{pb}$ ) of data in the muon (ditau) decay modes. The luminosity uncertainty cancels in the crosssection ratios, which provide a more precise test of Standard Model predictions and probe of parton density functions. All results are consistent with NNLO predictions. 

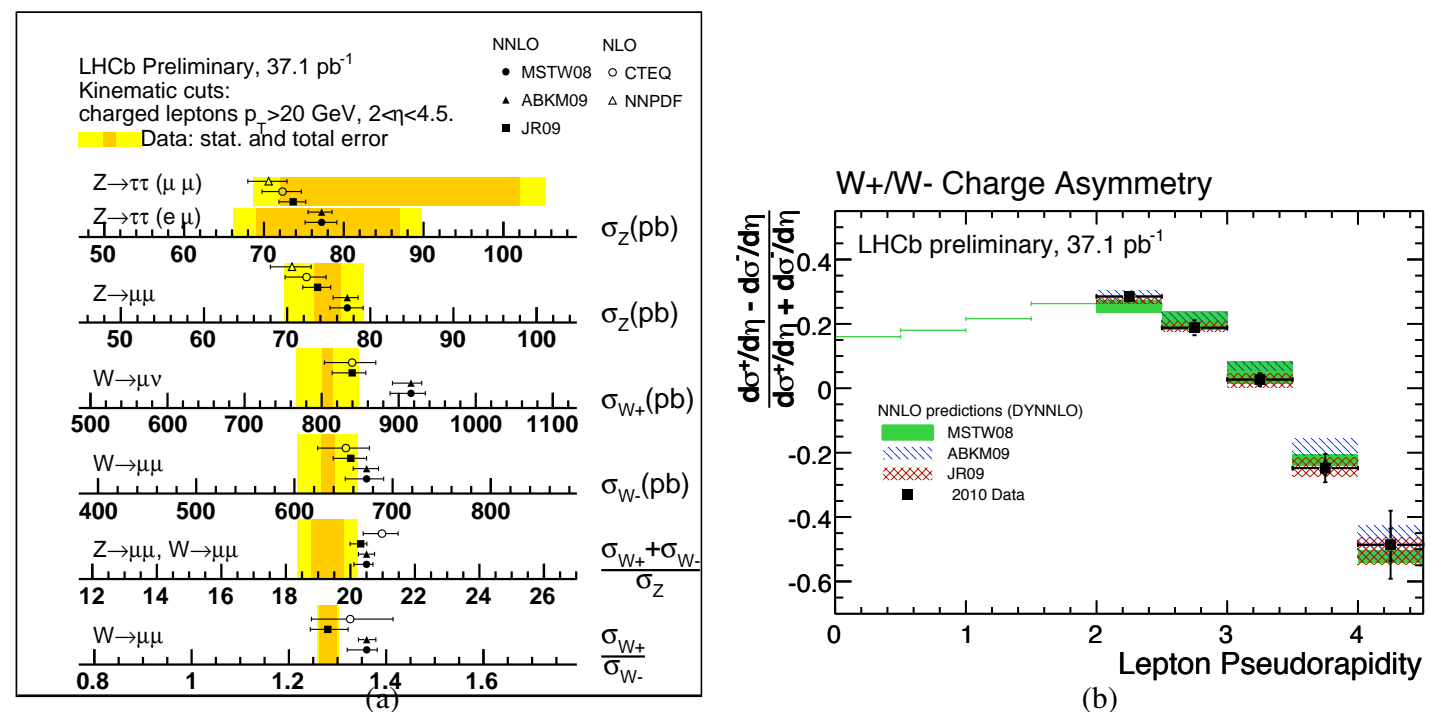

(b)

Figure 1: (a) $W$ charge asymmetry in bins of muon pseudo-rapidity compared to the NNLO prediction. The shaded and hatched areas represent the uncertainty arising from the PDF sets tested. The line represents the central value of the prediction for pseudorapidities below 2. (b) $Z, W+$ and $W$ - cross-section measurements and ratios (coloured bands) compared to NNLO and NLO predictions (points), for the PDF sets tested.

\section{References}

[1] See, for example, R. Thorne et al., arXiv:0808.1847 [hep-ph], published online under [doi:10.3360/dis.2008.30 ].

[2] A. Augusto Alves et al. JINST 3:S08005, 2008.

[3] The LHCb Collaboration, LHCb-CONF-2011-039.

[4] The LHCb Collaboration, LHCb-CONF-2011-041.

[5] P. Nason, JHEP 0411 (2004) 040, hep-ph/0409146; S. Frixione, P. Nason and C. Oleari, JHEP 0711 (2007) 070, arXiv:0709.2092;

S. Alioli, P. Nason, C. Oleari and E. Re, JHEP 1006 (2010) 043, arXiv:1002.2581.

[6] P. M. Nadolsky, H. -L. Lai, Q. -H. Cao, J. Huston, J. Pumplin, D. Stump, W. -K. Tung, C. -P. Yuan, Phys. Rev. D78 (2008) 013004. [arXiv:0802.0007 [hep-ph]].

[7] T. Sjostrand et al., Computer Phys. Commun. 135 238, 2001.

[8] C. M. Carloni Calame, G. Montagna, O. Nicrosini, M. Treccani, Phys. Rev. D69 (2004) 037301. [hep-ph/0303102].

[9] S. Van Der Meer, ISR-PO/68-31, 1968;

M. Ferro-Luzzi, CERN-PH-EP/2005-023 (2005). 